Old Dominion University

ODU Digital Commons

CCPO Publications

Center for Coastal Physical Oceanography

1993

\title{
A Comparison of Gulf Stream Sea Surface Height Fields Derived from Geosat Altimeter Data and Those Derived from Sea Surface Temperature Data
}

Tal Ezer

Old Dominion University, tezer@odu.edu

George L. Mellor

Dong-Shan Ko

Ziv Sirkes

Follow this and additional works at: https://digitalcommons.odu.edu/ccpo_pubs

Part of the Atmospheric Sciences Commons, Climate Commons, and the Oceanography Commons

\section{Repository Citation}

Ezer, Tal; Mellor, George L.; Ko, Dong-Shan; and Sirkes, Ziv, "A Comparison of Gulf Stream Sea Surface Height Fields Derived from Geosat Altimeter Data and Those Derived from Sea Surface Temperature Data" (1993). CCPO Publications. 119.

https://digitalcommons.odu.edu/ccpo_pubs/119

\section{Original Publication Citation}

Ezer, T., Mellor, G.L., Dong-Shan, K., \& Sirkes, Z. (1993). A comparison of Gulf Stream sea surface height fields derived from geosat altimeter data and those derived from sea surface temperature data. Journal of Atmospheric and Oceanic Technology, 10(1), 76-87. doi: 10.1175/1520-0426(1993)0102.0.CO;2 


\title{
A Comparison of Gulf Stream Sea Surface Height Fields Derived from Geosat Altimeter Data and Those Derived from Sea Surface Temperature Data
}

\author{
TAL EZER AND GEORGE L. MELLOR \\ Atmospheric and Oceanic Sciences Program, Princeton University, Princeton, New Jersey
}

DONG-SHAN KO AND ZIV SIRKES

Institute for Naval Oceanography, Stennis Space Center, Mississippi

(Manuscript received 5 March 1992, in final form 15 June 1992)

ABSTRACT

\begin{abstract}
Two types of satellite data, Geosat altimeter data and sea surface temperature data (SST), are compared and evaluated for their usefulness in assimilation into a numerical model of the Gulf Stream region. Synoptic sea surface height (SSH) fields are derived from the SST data in the following way: first, three-dimensional temperature and salinity analysis fields are obtained through the Optimum Thermal Interpolation System (OTIS), and then SSH fields are calculated using a primitive equation, free-surface, numerical model running in a diagnostic mode. The aforementioned SSH fields are compared with SSH fields obtained from the Geosat altimeter data. Use of Geosat data requires an estimate of the mean SSH field relative to the earth geoid. Three different methods to obtain the mean SSH field are demonstrated. The first method uses altimetry and SST data; the second uses a diagnostic calculation with climatological data; and the third uses prognostic numerical calculations. The three estimates compared favorably with each other and with estimates obtained elsewhere.

The comparison of the synoptic SSH fields derived from both data types reveals similarity in the Gulf Stream meanders and some mesoscale features, but shows differences in strength of eddies and in variability far from the Gulf Stream. Due to the smoothed nature of the OTIS analysis fields, the SSH derived from altimetry data has larger variability amplitudes compared to that derived from SST data.

The statistical interpolation method, which is used to interpolate altimetry data from satellite tracks onto the model grid, is also evaluated for its filtering effect and its sensitivity to different parameters. The SSH variability of the Gulf Stream was calculated from two years of the exact repeat mission of the Geosat satellite, where altimeter data were interpolated daily onto the model grid. It is suggested here that some of the underestimation of mesoscale variations by statistical interpolation methods, as indicated by previous studies, may be explained by the filtering effect of the scheme.
\end{abstract}

\section{Introduction}

It is evident that assimilation of data into numerical ocean models of the Gulf Stream will make important use of two distinctly different types of surface satellite data: multichannel sea surface temperature (MCSST) data and altimetry elevation data, or, in the present case, Geosat data. Each data type has errors that need to be evaluated before a continuous data assimilation can be used to produce a nowcast or forecast. For example, Geosat data is available only along satellite tracks that are separated by distances comparable to the dominant scales of the Gulf Stream system. On the other hand, SST data may be incomplete because of cloud cover. Since satellite data provide only surface information, this information must be projected into the deep ocean to obtain the three-dimensional oceanic

Corresponding author address: Dr. Tal Ezer, Atmospheric and Oceanic Sciences, P.O. Box CN710, Sayre Hall, Princeton University, Princeton, NJ 08544-0710. fields. This can be done, for example, by using "feature models" for SST data (e.g., Robinson et al. 1989) or by using surface-subsurface correlations (e.g., Mellor and Ezer 1991) for altimetry data. One of the main goals of this paper is to estimate errors associated with data and with analysis schemes and to provide perceptions for future data-assimilation studies.

Multichannel sea surface temperatures from satellite IR images provide global coverage of the World Ocean. The images can be used to obtain the Gulf Stream north wall location, subjectively or objectively. Previously, the former has been advantageous over the latter (Cornillon and Watts 1987), but recent improvement in the objective frontal-detection algorithms make the objective method almost as accurate as the subjective method (Cayula and Cornillon 1992). Surface meanders and ring paths obtained from the IR images can then be converted to subsurface information through the use of predetermined analytical formulas or "feature models" (e.g., Robinson et al. 1989), and be used for data assimilation into a dynamic ocean model. In 
this paper, the U.S. Navy's Optimum Thermal Interpolation System (OTIS), described by Clancy et al. (1990, 1992), is used to obtain three-dimensional analyses of temperature and salinity fields from subjective determination of the Gulf Stream edge and rings using MCSST information, together with expendable bathythermograph (XBT) data and climatology data for far fields. OTIS, which forms the basis of the Navy's daily analysis fields, is dominated, however, by Gulf Stream path and rings determined from the MCSST data and the feature model. The OTIS data is a major component in the Data Assimilation and Model Evaluation Experiments (DAMEE), a project organized by the Institute for Naval Oceanography. These data have been used to initialize and evaluate Gulf Stream models; the study of Ezer et al. (1992), for example, demonstrates that numerical models initialized with fields obtained from OTIS may have a forecast skill for at least a two-week period.

Satellite-derived altimetry data, such as those obtained from Geosat, provide a global coverage of the World Ocean, and are useful for studying sea surface variability (e.g., Le Traon et al. 1990). They can also be used, together with a numerical model and a dataassimilation scheme, to provide a nowcast of complete oceanic fields. Recent contributions to the science of altimetry data assimilation have been made, for example, by Holland and Malanotte-Rizzoli (1989), White et al. (1990), and Mellor and Ezer (1991). A problem associated with the use of altimetry data is that the relationship of the measured sea surface height to the geoid is unknown. Subtraction of the time-averaged altimetry data from the data of each satellite track removes the geoid, but the mean (over the record length) ocean circulation signal is removed as well. Glenn et al. (1991) used a numerical, quasigeostrophic ocean model updated with data to reconstruct the mean dynamic height relative to the geoid, while Kelly (1991) fitted along-track data to analytical formulas across the stream. Another way to estimate mean sea surface height is by dynamic height calculation (e.g., Levitus 1982 ) or by a diagnostic calculation (e.g., Mellor et al. 1982). In this paper we describe a somewhat different approach, using a limited number of altimetry and thermal analysis fields, to obtain the mean sea surface height field. The result is not greatly different from that obtained by Glenn et al. (1991) or Kelly (1991). Then, we combine the mean height field with the Geosat anomaly height fields to obtain Geosat (approximately) synoptic fields. These are compared with the corresponding height fields obtained from a diagnostic calculation that uses the three-dimensional OTIS temperature and salinity fields.

Since altimetry data is provided only along satellite tracks, data-assimilation schemes may use an optimal interpolation method (e.g., Mellor and Ezer 1991) to map the satellite data onto the model grid, using estimates of model and data errors, as well as other pa- rameters. Two types of this mapping (sometimes also called an objective analysis ) that have been used in ocean studies are statistical interpolation and dynamical interpolation (or assimilation): in the former, data are interpolated onto a standard (e.g., model) grid using only data statistics without any model calculation, while the latter uses model fields - and therefore model dynamics - as first-guess estimates for the assimilation process. The sea surface height (SSH) variability obtained from the two methods may be significantly different from each other; statistical assimilation usually underestimates mesoscale and small-scale variabilities (White et al. 1990; Mellor and Ezer 1991). It was believed to be due to the large separation distance between satellite tracks; that is, the model dynamics in the dynamical assimilation reconstructs the small-scale variability missed by the altimeter. Another factor, however, that may be responsible for reducing SSH variability inferred from statistical interpolation is the filtering effect of the interpolation scheme, which is studied here.

The data and the numerical model used for the diagnostic calculation are described in section 2 . In section 3 , we demonstrate a method to estimate mean height fields and then obtain synoptic height fields from the Geosat anomaly data. These are compared with synoptic elevation fields obtained from OTIS fields and the diagnostic calculation. In section 4 , the filtering effect of the statistical interpolation method is discussed. Section 5 concludes this study.

\section{Description of the data and the numerical model}

\section{a. Temperature and salinity fields inferred from sea surface temperature data}

Synoptic fields of temperature and salinity are obtained from OTIS. The global-scale system is described in detail by Clancy et al. (1990, 1992), and the regionalscale system (in particular, for the Gulf Stream region) is described by Cummings and Ignaszewski (1991). Minor modifications of the operational OTIS were adopted for the present study. We use the OTIS version 3 , implemented for DAMEE, a project organized by the Institute for Naval Oceanography. The first-guess fields are the monthly or seasonal climatologies obtained from the U.S. Navy's General Digital Environmental Model (GDEM) described in the following. Then satellite IR images are used to subjectively (by Dr. Scott Glen) produce "bogus maps" of the Gulf Stream north wall and ring locations and size; a feature model projects the surface information into the deep layers, producing three-dimensional synthetic temperature and salinity fields. The synthetic fields, the MCSST used directly at the surface, the GDEM climatology, and XBT data are used to produce threedimensional thermal fields via an optimal interpolation method. Some Geosat tracks are used to help determine the edge of the stream in cases of cloud cover, for ex- 
ample; thus, the OTIS fields are not totally independent from the Geosat data. As will be shown later, the resultant OTIS fields, however, are dominated by the feature model. The data is projected onto a $0.2^{\circ} \times 0.2^{\circ}$ horizontal grid and 34 vertical levels.

The annual climatology used here was obtained from the GDEM, developed and maintained by the Naval Oceanographic Office. It uses observed temperature and salinity data from the Master Oceanic Observation Data Set (MOODS), developed and maintained by the U.S. Navy's Fleet Numerical Oceanography Center (FNOC); monthly climatologies are used for the upper $400 \mathrm{~m}$, and seasonal climatologies are used for the deeper levels. The data are projected onto a $0.5^{\circ} \times 0.5^{\circ}$ horizontal grid and on 30 vertical levels.

\section{b. The Geosat altimetry data}

The altimetry data used here is from the exact repeat mission of the geodetic satellite, Geosat, covering a twoyear period from November 1986 to November 1988. The data are available approximately every $7 \mathrm{~km}$ along each track, which repeat every 17.05 days. In the modeled area there are typically two tracks per day. A new set of estimated Geosat orbits for the exact repeat mission was obtained by Haines et al. (1990). Quality control and editing were applied to this dataset, and long-wavelength periodic orbit errors, found from spectral analysis (Sirkes and Wunsch 1990), were removed from the data. For the Gulf Stream region, all tracks within $20^{\circ}-50^{\circ} \mathrm{N}$ and $40^{\circ}-80^{\circ} \mathrm{W}$ and where water depth is greater than $1000 \mathrm{~m}$ were used. In this area, the mean elevation along each track is obtained from all available repeat cycles and removed from the individual repeat cycles. Therefore, the geoid is completely removed, as well as the mean ocean circulation signal: here these data are called the Geosat anomaly SSH data or, simply, the Geosat data.

\section{c. The numerical ocean model}

Since the emphasis in this paper is on data sources and interpolation schemes, the numerical model is described only briefly; the model will primarily be used diagnostically (temperature and salinity held constant to obtain sea surface heights from the temperature and salinity fields). The model is the three-dimensional, free-surface, Princeton primitive equation ocean model clescribed by Blumberg and Mellor (1987). It has bottom-following vertical sigma coordinates (with 20 levels in this application) and a curvilinear orthogonal, coastal-following, horizontal grid with a typical resolution of $10-18 \mathrm{~km}$ in the Gulf Stream region (see Fig. 1 for the grid and the bathymetry). The model has been used for many applications such as estuaries, bays, and coastal oceans. In the Gulf Stream region, the model has been used before for data assimilation studies (Mellor and Ezer 1991; Ezer et al. 1991), for Gulf
Stream separation and surface-forcing sensitivity studies (Ezer and Mellor 1992), for studying the interaction of the Gulf Stream with the coastal ocean (Oey et al. 1992), and for evaluation of the forecast skill of the model (Ezer et al. 1992).

In the present application, all of the OTIS and GDEM data are presented on the model grid (Fig. 1), where bilinear and cubic-spline schemes were used in the horizontal and in the vertical direction, respectively, to interpolate the data onto the irregular model grid. The Geosat data are interpolated from satellite tracks onto the model grid using the interpolation method described in the following.

\section{Derivation of mean and synoptic sea surface height fields}

\section{a. Estimating the mean sea surface height}

Here we calculate the mean dynamic height so that synoptic realizations of sea surface height can subsequently be calculated. For each time $t_{m}$ we form an independent estimate of the mean Geosat elevation field $\eta^{G}\left(x, y, t_{m}\right)$ using the OTIS field $\eta^{o}\left(x, y, t_{m}\right)$ and the Geosat anomaly field $\delta \eta^{G}\left(x, y, t_{m}\right)$ according to

$$
\begin{aligned}
\eta^{\circ}\left(x, y, t_{m}\right)=\eta^{G} & \left(x, y, t_{m}\right) \\
& +\delta \eta^{G}\left(x, y, t_{m}\right)+\varepsilon\left(x, y, t_{m}\right),
\end{aligned}
$$

where $\varepsilon$ represents errors in the data and in the processing procedures and, obviously, the fact that OTIS mean heights may differ from the Geosat mean heights. In the hypothetical case that the errors are null, only one OTIS and one Geosat realization would be required; thus, the mean SSH will be simply $\eta^{o}-\delta \eta^{G}$. In fact, we will determine nine independent estimates of the mean elevation field and then average them to reduce the error.

Synoptic temperature and salinity fields were obtained from OTIS 3 for several periods. In areas where cloud cover prevents an accurate determination of the Gulf Stream axis, images from previous or future dates are used. After the OTIS data were interpolated into the model grid, the numerical model diagnostically calculated the corresponding OTIS surface elevation $\eta^{o}\left(x, y, t_{m}\right)$. Nine such fields were obtained this way for $t_{m}=6,13,20$ May 1987; 7, 14, 21 July 1987; 4, 11, 18 May 1988. (These dates were chosen because of the relatively good coverage of IR images over the region at these periods.) For each of these dates, the Geosat SSH anomaly at a model grid point $i$ is obtained from

$$
\delta \eta_{i}^{G}=\sum_{\alpha=1}^{N} P_{i \alpha} \delta \eta_{\alpha}^{G},
$$

where the subscript $\alpha$ represents data-point location in space and time centered about $t=t_{m}$. The weights $P_{i}$ are determined from the interpolation scheme de- 


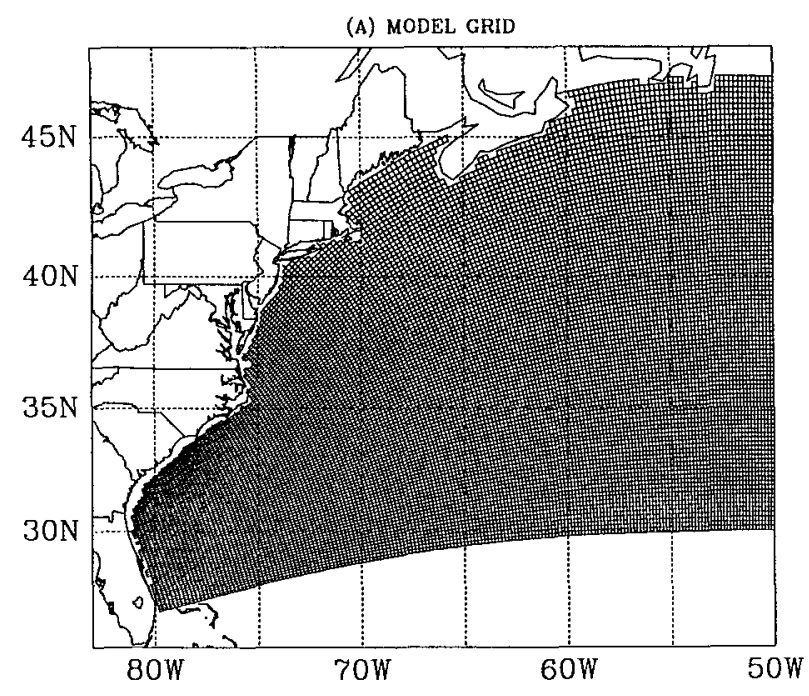

(B) BOTTOM TOPOGRAPHY

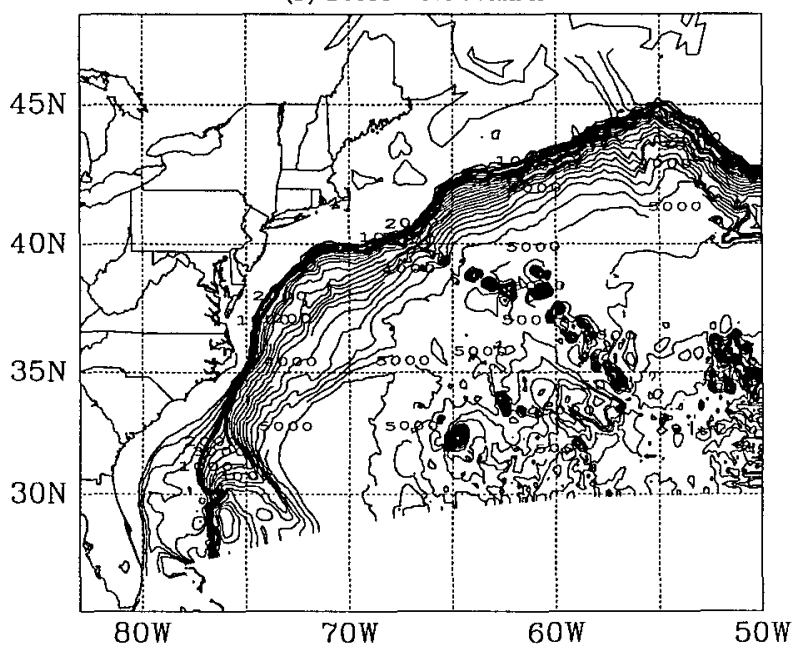

FIG. 1. (a) The curvilinear orthogonal model grid. (b) The bottom topography of the model; the contour interval is $200 \mathrm{~m}$.

scribed in the following. The $N$ data points used in the interpolation for each model grid point are the ones having the highest correlation in space and time with the model point, using a normalized autocorrelation function of the form

$$
G_{\alpha \beta}=\exp \left[-\left(k_{x} \Delta x\right)^{2}-\left(k_{y} \Delta y\right)^{2}-\left(k_{t} \Delta t\right)^{2}\right],
$$

where the time and length scales, $k_{x}^{-1}=k_{y}^{-1}=170 \mathrm{~km}$ and $k_{t}^{-1}=22$ days, are estimated from model statistics (Mellor and Ezer 1991) and the $\Delta$ 's represent the difference in time and space between the model grid and the data points. The data points were chosen from a window of 17 days (i.e., one repeat Geosat cycle) centered around each OTIS date. In the experiments described here we take $N=12$. (The sensitivity of the scheme to the value of $N$ is evaluated later in section
4.) The weights $P_{i \alpha}$ are calculated by solving the matrix equation

$$
\sum_{\alpha=1}^{N} P_{i \alpha} G_{\alpha \beta}=G_{i \beta} \quad \beta=1, \cdots, N .
$$

Note that in the case of continuous data assimilation, where model and data errors are to be taken into account, an additional term, representing the data error, is added to the left side of (4). Finally, we estimate the mean SSH in (1) by

$$
\overline{\eta(x, y)}=\frac{1}{M} \sum_{m=1}^{M}\left[\eta^{o}\left(x, y, t_{m}\right)-\delta \eta^{G}\left(x, y, t_{m}\right)\right] \text {, }
$$

where $M=9$ in our case. Of course, if $M$ is large enough, this mean will be the same as the mean over the OTIS SSH fields since the mean of the Geosat anomaly is zero by definition: the foregoing procedure simply reduces the net error due to the small sample size. Mean SSH $\overline{\eta(x, y)}$ is plotted in Fig. $2 \mathrm{a}$ and is in good agreement with fields obtained by Kelly (1991) and Glenn et al. (1991). Two alternative methods of estimating the mean SSH field are also presented in the following.

The mean SSH field also can be estimated through a diagnostic calculation using the numerical model and annual climatological temperature and salinity fields from GDEM. The model, after running in a diagnostic mode for 10 days, produced surface elevation plotted in Fig. 2b. The smoother and wider Gulf Stream reflects the correspondingly smooth GDEM climatology. It is interesting to compare between the mean SSH obtained from the diagnostic calculation and the one obtained from a simpler method, a dynamic height calculation, using the same GDEM data. The results (not shown) show that the diagnostic and dynamic-height calculations are virtually identical if the level of no motion in the latter is chosen to be $3000 \mathrm{~m}$; if the level of no motion is at $1000 \mathrm{~m}$, the resultant SSH gradient across the Gulf Stream is reduced by approximately $10 \%$. In the present application we prefer to use the diagnostic model since it yields an estimate of elevation in shallow waters, whereas the dynamic-height calculation does not.

Although it will play no further role in this paper, it is nevertheless of singular interest to compare the foregoing estimates of the mean SSH field with the estimate obtained from a prognostic calculation of the numerical model (temperature and salinity are advected and diffused vertically and horizontally). Figure $2 \mathrm{c}$ shows the mean SSH field calculated from a oneyear model simulation. The model was initialized with synoptic OTIS field and forced by monthly climatologies of wind-stress and heat-flux data [see Ezer and Mellor (1992) for more detailed description of the surface forcing] but without any data assimilation. The model mean SSH field (Fig. 2c) compared well with the field obtained from data (Fig. 2a); however, the 
(a)

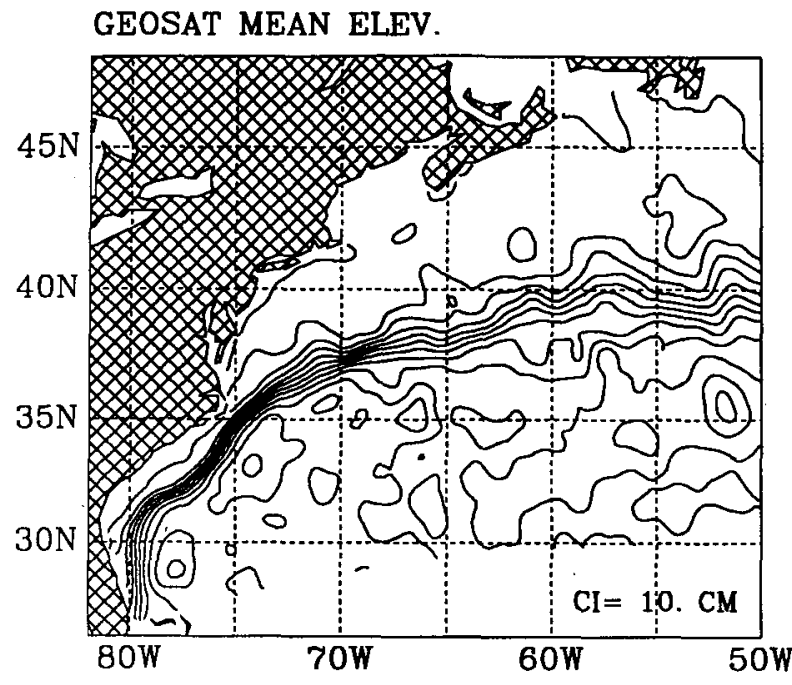

(c)

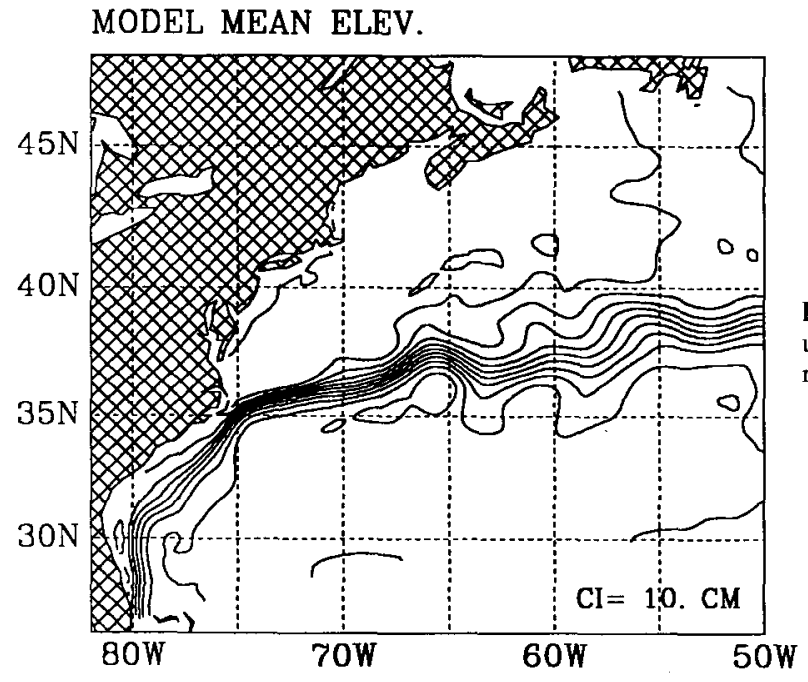

(b)

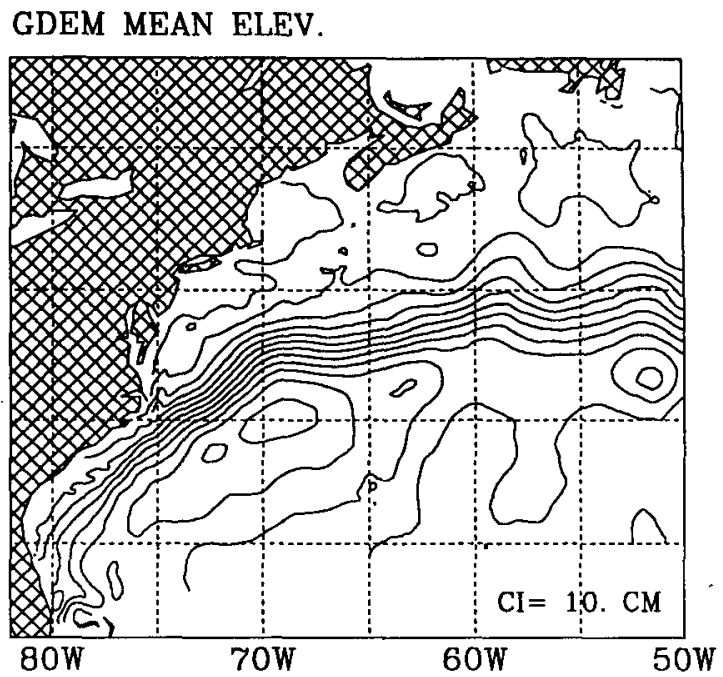

FIG. 2. Surface elevation climatology obtained from (a) Eq. (5) using OTIS and Geosat data, (b) diagnostic calculation using GDEM climatology data, and (c) a one-year prognostic run of the numerical model.

modeled mean Gulf Stream axis is somewhat more southward than the observed one.

\section{b. Estimating synoptic sea surface height fields}

Now, the synoptic SSH fields can be constructed by adding the Geosat-derived SSH anomaly from (2) of each of the nine cases to the mean SSH obtained from (5). The SSH fields obtained from Geosat and those obtained from diagnostic calculation using OTIS data, are shown in Figs. 3a, 3b, and 3c for the May 1987, July 1987, and May 1988 cases, respectively. Also shown are the absolute differences between each set of fields (area where the differences greater than $0.2 \mathrm{~m}$ are shaded ) and the 17-day satellite tracks used in the altimetry interpolation. [Note, for example, that during the last period of the Geosat mission (Fig. 3c) more data is missing than in the first period (Fig. 3a).] We observe that there are significant similarities and some differences between the OTIS and the Geosat fields.

Since we do not know the SSH fields of the real ocean, we can only speculate on possible errors in each data type. Errors in the OTIS-derived fields are due to errors in determining the Gulf Stream and ring locations from IR images and from the feature model scheme. Cornillon and Watts (1987) estimated the error of subjective determination of the Gulf Stream edge to be approximately $15 \mathrm{~km}$. Their study, however, was conducted west of $70^{\circ} \mathrm{W}$ where the variability is much smaller than in the eastern portion of the Gulf Stream, where it passes near the New England seamounts; thus, larger errors are expected in our case. The use of the 
GEOSAT ELEV.

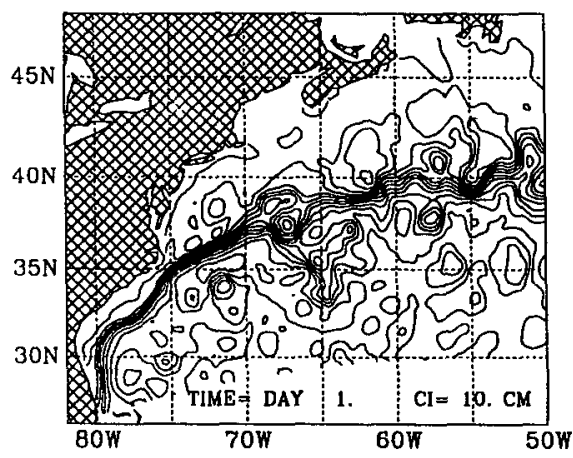

OTIS ELEV.

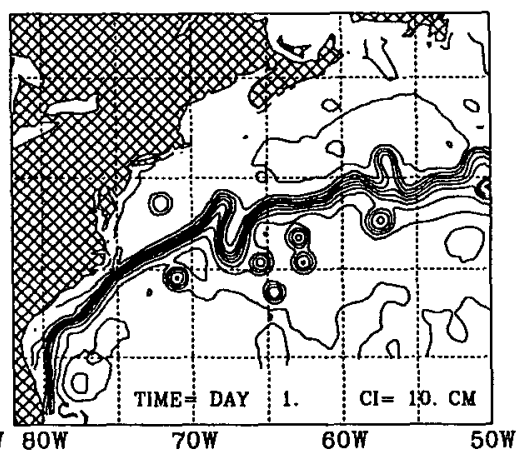

OTIS ELEV.
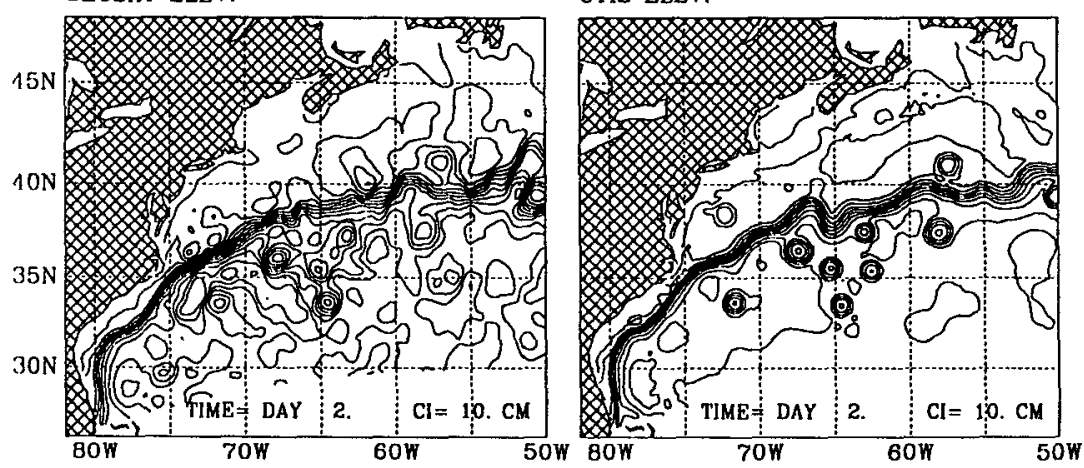

OTIS ELEV.
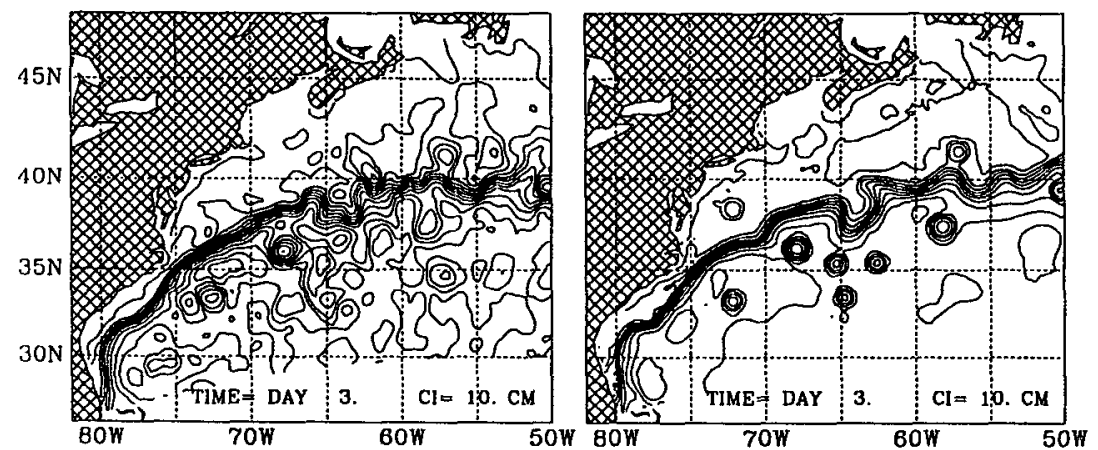

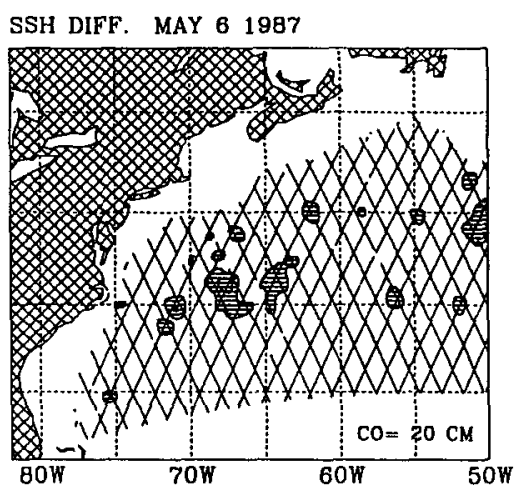

SSH DIFF. MAY 131987

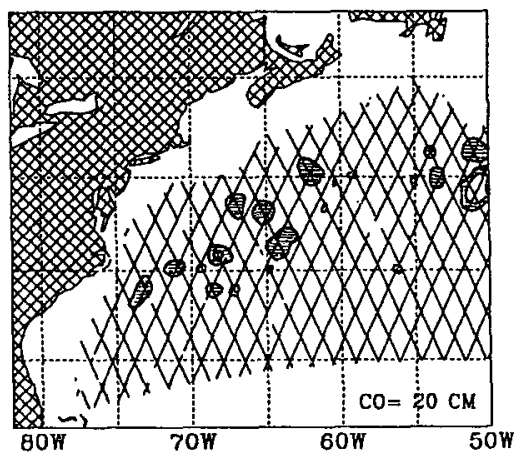

SSH DIFF. MAY 201987

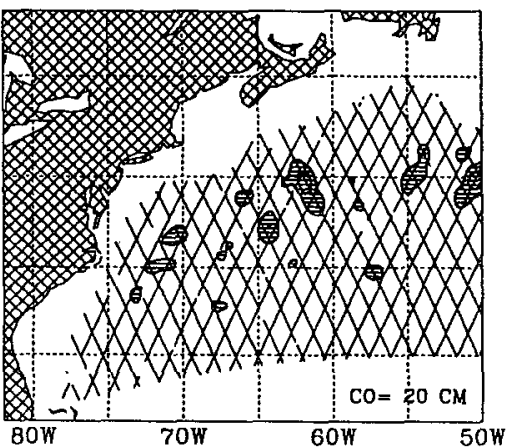

FIG. 3a. SSH obtained from the statistical interpolation method, using 17 days of Geosat altimetry data and climatology from Eq. (5) (left panels); SSH obtained from diagnostic calculations using OTIS temperature and salinity data fields (middle panels), absolute difference between the OTIS and the Geosat fields (shaded area represents difference greater than $0.2 \mathrm{~m}$ ), and satellite tracks used (right panels). From top to bottom are 6,13, and 20 May 1987.

feature model in the OTIS calculation may introduce additional errors in the projection of the surface information into the subsurface layers. Errors in the Geosat-derived fields are probably due to altimetry noise (about $0.05 \mathrm{~m}$ ), the interpolation into regions between tracks where data is not available, and errors in the mean SSH that was calculated. Since data over 17 days is used in the interpolation, time-dependent variations may be translated into spatial errors in the SSH fields.

Generally, the Geosat fields have more small-scale variations far from the stream, where OTIS fields are set to climatological values. On the other hand, some eddies cannot be resolved by the Geosat coverage of tracks. Some examples of the differences follow. On 6 May 1987 (Fig. 3a), the meander near $67^{\circ} \mathrm{W}$ seen in 

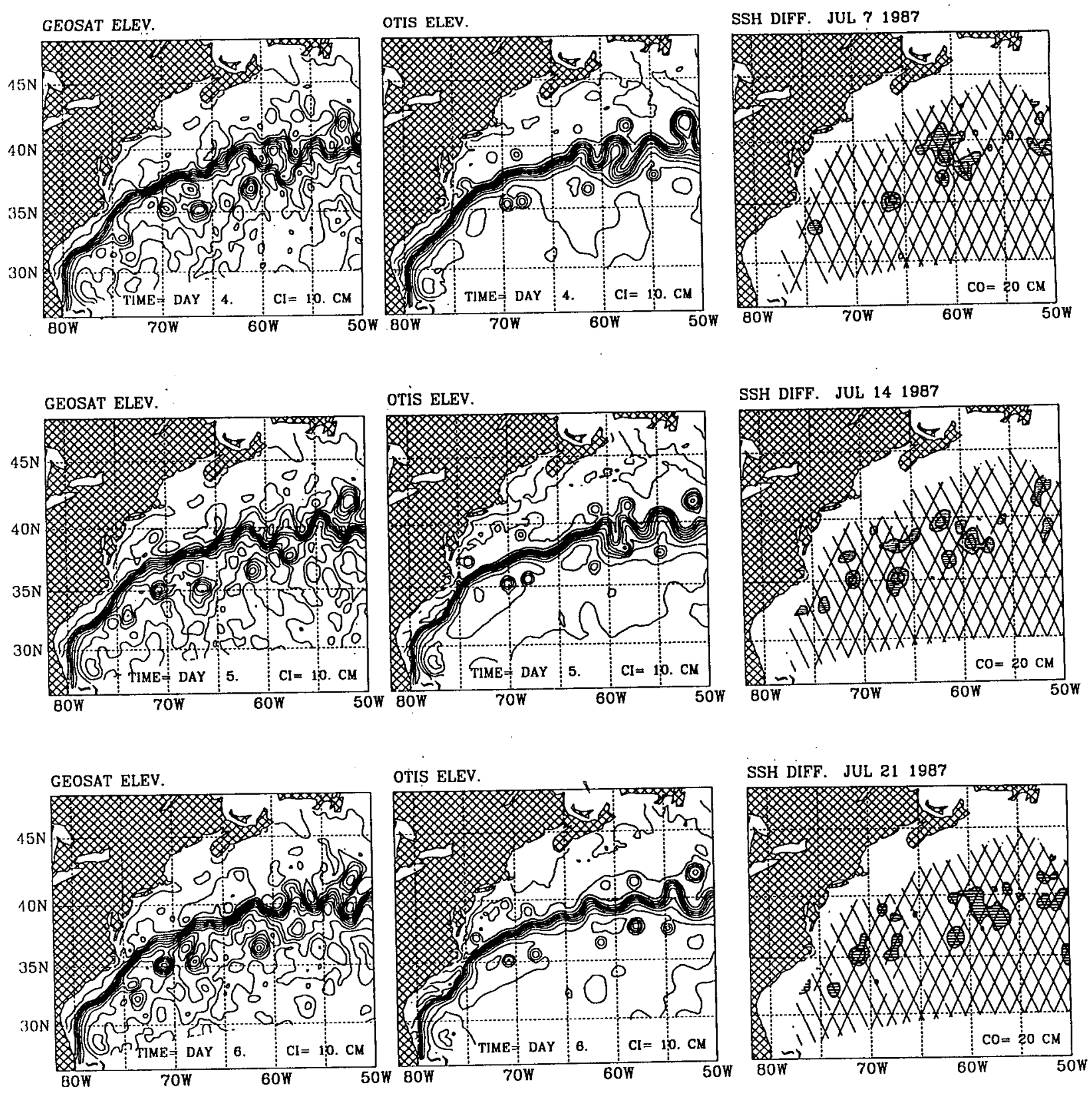

FIG. 3b. Same as Fig. 3a but for 7, 14, and 21 July 1987.

the OTIS field is not well resolved by the Geosat data; however, the location and strength of the cold-core ring developed from this meander, which can be seen on 13 and 20 May 1987, are in good agreement between the two fields. Note that while the interaction of a coldcore and a warm-core ring with the stream at $58^{\circ} \mathrm{W}$ is well defined by both fields, the cold-core ring at $34^{\circ} \mathrm{N}$, $57^{\circ} \mathrm{W}$ is missing from the OTIS field (lower panel of Fig. 3a). In the July 1987 case, note, for example, the differences in strength and location of the three cold rings between $60^{\circ}$ and $75^{\circ} \mathrm{W}$ (Fig. 3b). In the May
1988 case, altimetry data along several tracks are missing, and as a result, differences are larger there (right panels of Fig. 3c). The large meander crest at $65^{\circ} \mathrm{W}$ on 4 May 1988, which separated into a warm-core eddy observed on 18 May 1988 , is well resolved by both fields, but is stronger in the Geosat-derived SSH field (Fig. 3c). The meanders at $62^{\circ} \mathrm{W}$ on 18 May 1988 , however, are not well resolved by the Geosat data.

Note that in areas where Geosat data is not available (e.g., the shallower South Atlantic bight) the elevation fields are identical to $\overline{\eta(x, y)}$. Note also that the SSH 

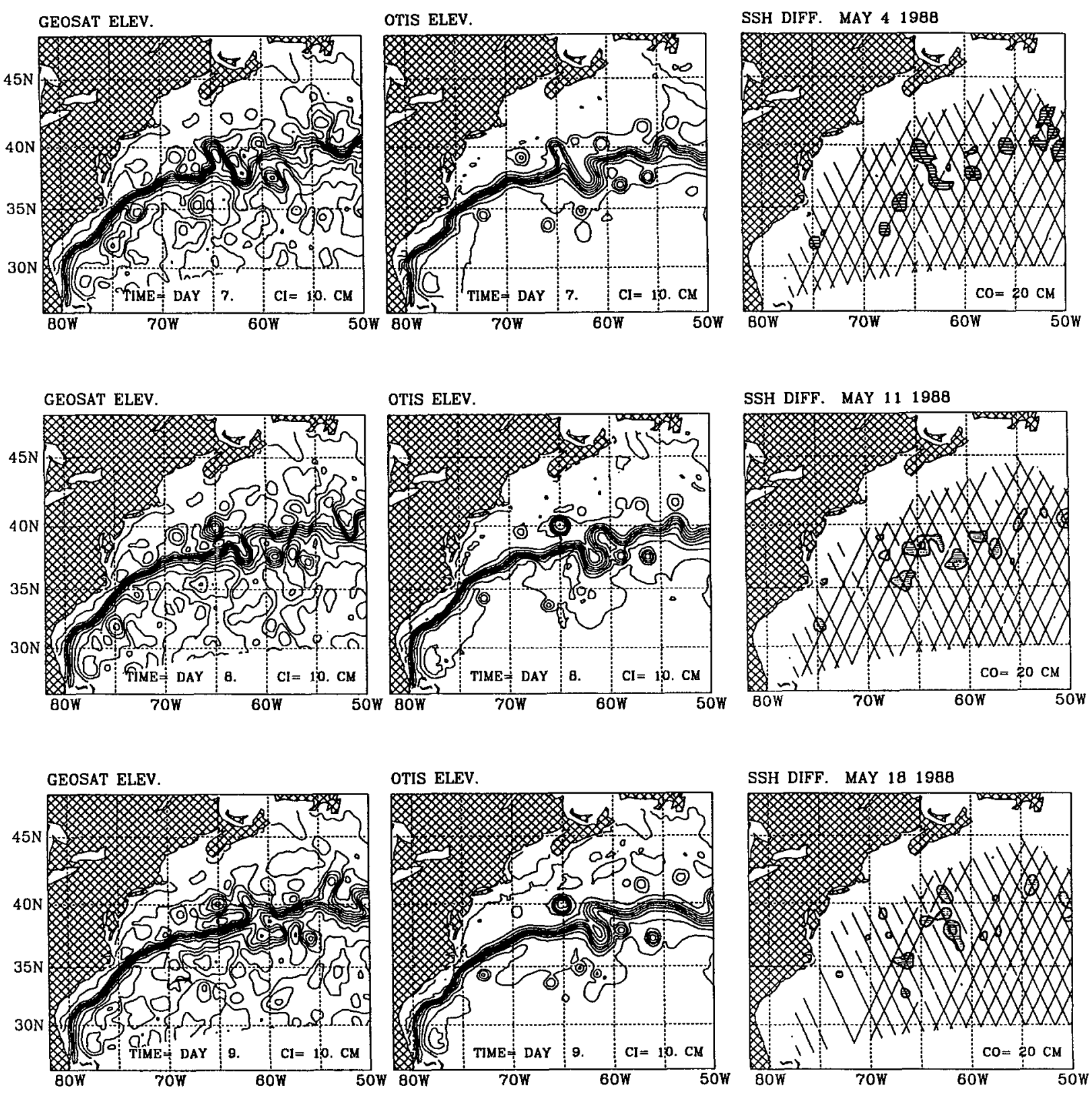

Fig. 3c. Same as Fig. 3a but for 4, 11, and 18 May 1988.

gradient across the stream is more uneven in the Geosat fields, with larger gradients in areas where Geosat tracks are crossing the stream. This bias is expected since, far from data points, the analysis fields are smoothed more by the interpolation scheme.

\section{c. Statistical comparison of synoptic sea surface height fields}

After the qualitative comparison described in the preceding section, a more quantitative evaluation of the statistical characteristics of the Geosat- and the
OTIS-derived elevation fields are presented, using three statistical measures: rms error, anomaly-pattern correlation, and error in Gulf Stream axis. Suppose we would like to compare two elevation fields $\eta^{a}(x, y)$ and $\eta^{b}(x, y)$, the rms error between the two fields is given by

$$
\mathrm{ER}^{a b}=\left\langle\left(\eta_{i}^{a}-\eta_{i}^{b}\right)^{2}\right\rangle^{1 / 2},
$$

where the angular brackets represent an area average. The superscripts $a$ and $b$ represent OTIS and Geosat fields, respectively (i.e., ER is the rms error between 
the two), or they represent the same data type (OTIS or Geosat) but for fields that are separated by a time lag $\tau$ (i.e., as $\tau \rightarrow \infty$ ER represents the natural variability of each data type). The total area $A$ in which the rms error is calculated is chosen here as the region north of $35^{\circ} \mathrm{N}$ and for water depths greater than 2000 $\mathrm{m}$; that is, regions with least variability, such as the subtropical gyre and the continental shelf, are neglected here. Another measure, the anomaly-pattern correlation, is calculated according to

$$
\mathrm{AC}^{a b}=\frac{\left\langle\left(\eta_{i}^{a}-\left\langle\eta^{a}\right\rangle\right)\left(\eta_{i}^{b}-\left\langle\eta^{b}\right\rangle\right)\right\rangle}{\left[\left\langle\eta_{i}^{a}-\left\langle\eta^{a}\right\rangle\right\rangle^{2}\left\langle\eta_{i}^{b}-\left\langle\eta^{b}\right\rangle\right\rangle^{2}\right]^{1 / 2}}
$$

While (6) represents the average difference in SSH between the two fields and is 0 for identical fields, (7) represents the correlation between deviation patterns and ranges between -1 and 1 and is unity for identical fields.

We also calculate the error in the Gulf Stream axis, AXERR, which is defined as the area between two stream axes (derived from the SSH contour representing the maximum gradient of surface elevation across the stream) divided by the length of the path.

The error between the Geosat and the OTIS elevation fields is defined as the interpolated altimetry field at time $t$, compared to the corresponding diagnostically adjusted OTIS elevation. (If the OTIS fields accurately represent the ocean, then this error would be the "nowcast" error of the Geosat realizations.) We also calculate the difference between an initial field (in this case the first day of each of the three cases) and the following fields as a function of a time lag. The latter is called the persistence error, which is often used to evaluate nowcast-forecast skills; it represents the natural variability of the data.

First, we discuss the persistence errors of the three statistical measures: ER, AXERR, and AC are plotted as a function of time lag in Figs. 4a-c, respectively (small "O" and "+" marks represent the OTIS and the Geosat data, respectively, for different dates). Since the typical time scale for mesoscale events in the Gulf Stream region is approximately $20-40$ days, after a long enough time, the persistence errors reach a constant level, representing the relation between two independent realizations. For a large time lag, only the ER shows significant difference between the OTIS and the Geosat fields (Fig. 4a). This indicates that the natural variability in anomaly pattern correlation and in the Gulf Stream axis is similar for both OTIS and Geosat, and only the eddy field is different. Note that the natural variability in the OTIS fields is considerably smaller than the one obtained from Geosat (maximum rms error of approximately $0.18 \mathrm{~m}$ for the OTIS compared to $0.26 \mathrm{~m}$ for the Geosat). This results from the smoothed nature of the OTIS system and the relatively noisy Geosat data. Some of the Geosat "noise," how-

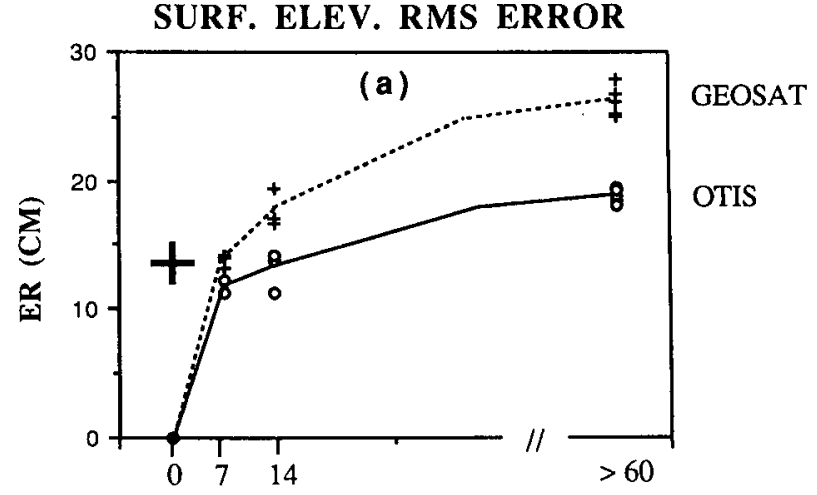

GULF STREAM AXIS ERROR

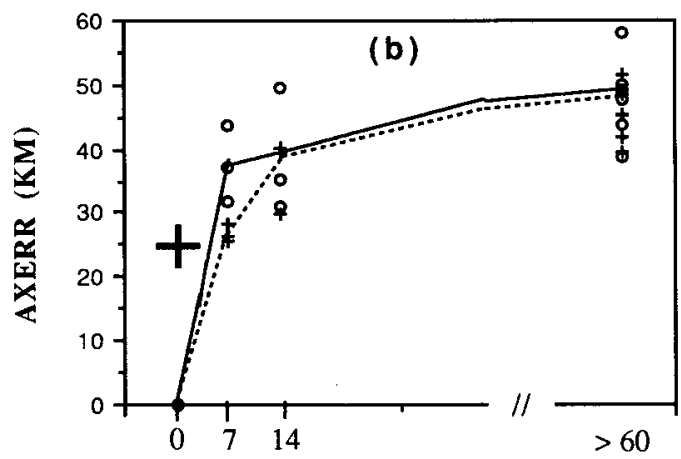

SURF. ELEV. ANOMALY CORR.

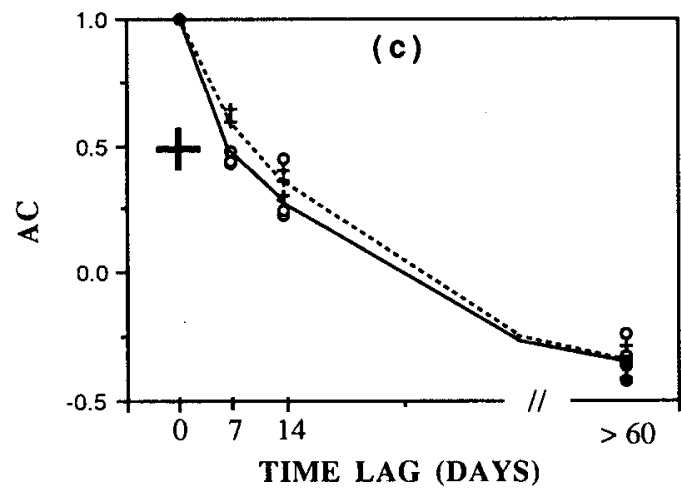

FIG. 4. Persistence errors in SSH fields as a function of time lag for OTIS data (small " $O$ " marks indicate individual dates and solid. lines indicate averages) and for Geosat data (small "+" marks and dotted lines). A large " + " at zero lag represents the average error between the OTIS and the Geosat fields (calculated from the nine cases). (a) Root-mean-square error, ER; (b) axis error, AXERR; and (c) anomaly pattern correlation, AC.

ever, is real; the oceans do have a large range of smallscale spatial variations that are clearly missing from the OTIS data. For example, the Geosat-derived fields in Fig. 3 indicate several recirculation gyres north and south of the stream; similar features are obtained from the numerical simulations of Ezer and Mellor (1992) and from observations. 
We now evaluate the average differences between the OTIS- and the Geosat-derived fields (calculated by averaging the differences of the nine cases using the same three measures as before); they are indicated in Fig. 4 by the large "+" marks at $\tau=0$. The average rms difference between the OTIS and the Geosat fields is approximately $0.14 \mathrm{~m}$, about half of the maximum Geosat persistence error (Fig. 4a). The average AXERR is about $25 \mathrm{~km}$ compared to approximately 50 $\mathrm{km}$ of the maximum persistence error (Fig. $4 \mathrm{~b}$ ). There is also an average bias of $19 \mathrm{~km} \pm 13 \mathrm{~km}$ between the mean Geosat and the mean OTIS axis. This southward bias of the OTIS axis due to its feature model is consistence with Glenn's finding (personal communication). The anomaly correlations between the OTIS and the Geosat fields are on average approximately 0.5 (with $90 \%$ confidence level, based on the Student's ttest) (Fig. 4c). These systematic differences should be taken into account when both data types are used for data assimilation.

\section{The effect of statistical interpolation on the sea surface height variability}

The area-averaged surface-elevation rms (representing the SSH variability) obtained from statistical interpolation of Geosat-type data (i.e., a continuous interpolation of data onto a model grid without dynamic calculation ) may be smaller than that of a numerical model or that of a dynamic assimilation (White et al. 1990; Mellor and Ezer 1991). There are two possible reasons for this. First, because of the Geosat track schedule and, especially, the distance between tracks, small-scale oceanic features cannot be resolved. Second, the interpolation method that is used to map data from satellite tracks onto the model grid acts as a filter that may reduce the total variability. We study here the latter effect, as a function of some of the interpolation parameters. In a continuous statistical interpolation the SSH anomaly analysis at a given time and space (on model grid points) is obtained from

$$
\delta \eta_{i}^{t+\Delta t}=\delta \eta_{i}^{t}+\sum_{\alpha=1}^{N} P_{\alpha}^{t}\left(\delta \eta_{o \alpha}^{t}-\delta \eta_{i \alpha}^{t}\right)
$$

where subscript $o$ represents the Geosat data and $\Delta t$ is the interpolation time step. If we use each observed data only once (e.g., whenever there is a satellite track over the model area) and if, at this time, we neglect measurement error, (3) and (4) can be used to calculate the weights. We have done this calculation for the two years of the Geosat data (November 1986-November 1988 ), using $\Delta t=1$ day (i.e., interpolating at each day all the data available in a 1-day window), saving the SSH fields every day. Note that the SSH variability calculated from these daily fields is independent of the initial field used in (8). Unlike the calculations of sec- tion 3, where data from past and future time are used, only present data are used here at each day, as in a more realistic operational scenario and as was done in the statistical interpolation section of the study of Mellor and Ezer (1991).

Two of the parameters that may affect the SSH variability are the number of points $N$ used to update each model point and the length scales $k_{x}^{-1}, k_{y}^{-1}$. When us-
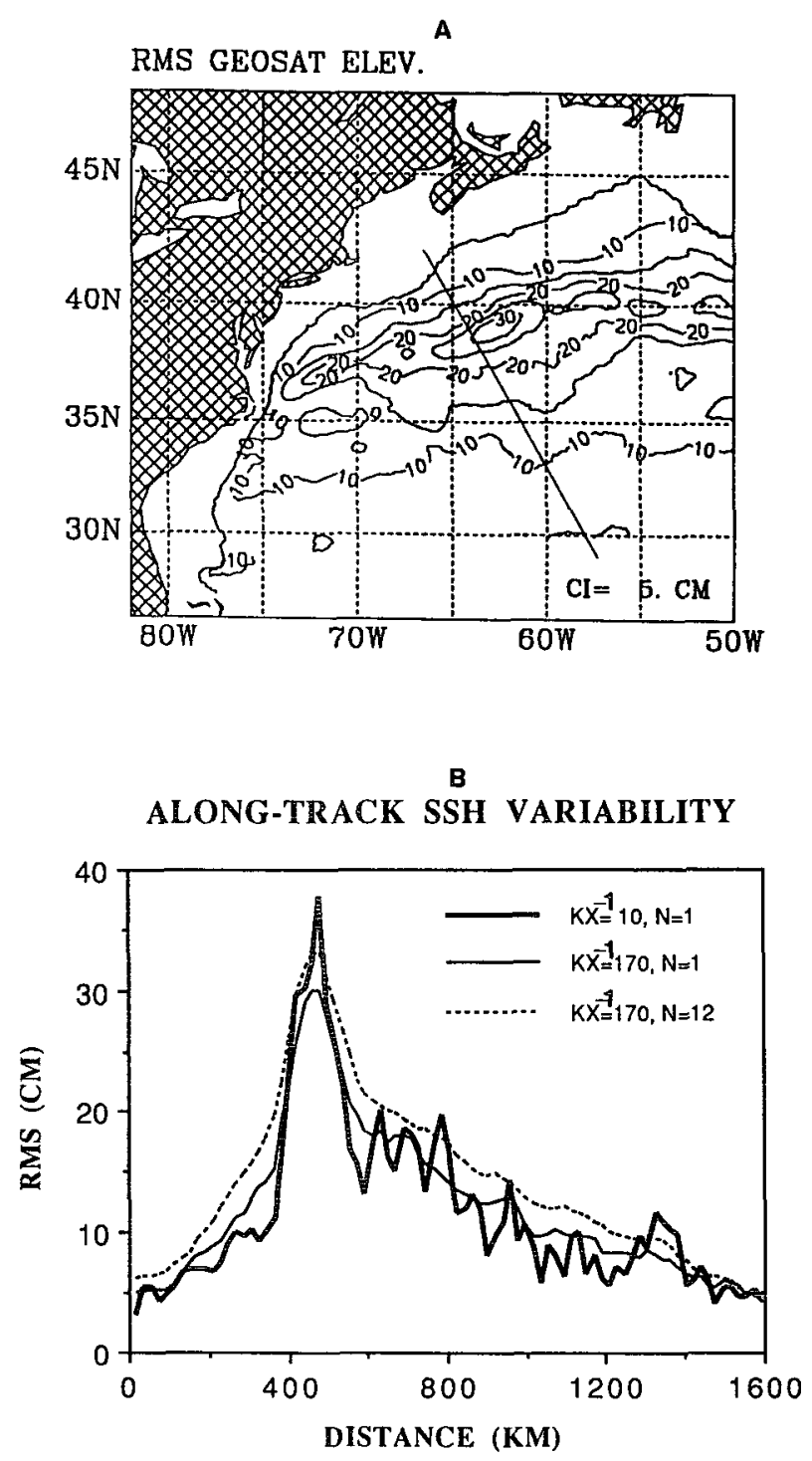

FIG. 5. (a) The rms of surface-elevation anomaly obtained from two years of Geosat altimetry data interpolated daily onto the model grid (interpolation parameters: $N=12$ data points per model grid point; length scale $k_{x}^{-1}=k_{y}^{-1}=170 \mathrm{~km}$ ). The contour interval is $0.05 \mathrm{~m}$. The shoreward-most line is the $1000-\mathrm{m}$ isobath contour; altimetry data were used for deeper regions only. (b) A cross section of the rms of SSH along the track indicated in (a). The different values of $N$ and $k_{x}^{-1}=k_{y}^{-1}$ are indicated. The wide solid line represents the closest estimate of the SSH variations obtained directly from the altimeter without the statistical interpolation. 
ing a realistic length scale of $170 \mathrm{~km}$, the maximum $\mathrm{rms}$ of SSH anomaly is $0.3 \mathrm{~m}$ for $N=1,0.33 \mathrm{~m}$ for $N$ $=4$, and $0.34 \mathrm{~m}$ for $N=8$ or $N=12$. Figure 5a shows an example of the rms anomaly; all these cases look similar to each other, so only one is shown here (increasing $N$ above 6 makes insignificant difference). Note the three maxima along the stream west of the New England seamounts; similar features, but with fewer details, are given, for example, by Le Traon et al. (1990) and Kelly (1991).

The SSH variability is more sensitive, however, to changes in the length scale. For example, for $N=1$ and when $k_{x}^{-1}$ decreases from $170 \mathrm{~km}$ to $10 \mathrm{~km}$, the maximum rms increases from 0.30 to $0.39 \mathrm{~m}$. Decreasing the length scale to such a small value, however, is impractical for data-assimilation applications since variability away from tracks decreases: when contouring variability, a checkerboard-like pattern is obtained. Note that if $N=1$ and the length scale is small enough, the variability on model points that fall directly, or are very close to satellite tracks, is almost the same as the variability obtained directly from the altimetry data without the optimal interpolation. Figure $5 b$ shows the two-year rms along one satellite track for different parameters. Increasing the length scale smoothes the SSH variability and cuts some of the maxima peaks. The amplitude of some of the peaks, however, is only slightly above the noise level of the Geosat data $(\sim 0.05$ $\mathrm{m})$. On the other hand, increasing $N$ increases the total level of rms since contribution from more points is allowed. For our model grid resolution, which is in average about twice the Geosat resolution along tracks $(\sim 7 \mathrm{~km})$, using more than $N=6$ data points to update each model grid point does not make a significant difference. Therefore, from these experiments it seems that there can be as much as $30 \%$ reduction in the SSH variability obtained by the statistical interpolation method, although the magnitude of this reduction is usually smaller than $30 \%$ and depends on the length scale used in the interpolation. An additional reduction of variability, when statistical assimilation is compared to dynamic assimilation, is due to small-scale variabilities that cannot be resolved by the satellite-track schedule (White et al. 1990; Mellor and Ezer 1991).

\section{Conclusions}

Synoptic SSH fields have been obtained from two satellite data sources: Geosat altimetric data, and SST data. The SSH fields derived from the altimetry data use the statistical interpolation method to map data from satellite tracks onto the model grid; the SSH fields from SST data involves determination of the Gulf Stream north wall and ring locations, using the thermal analysis interpolation system, OTIS, and diagnostic model calculations. Examination of Fig. 3 shows that mesoscale variability from the two determinations is in fair agreement, although there are obvious differences. While OTIS data neglect small-scale variability far from the Gulf Stream and may be subject to errors in the projection of surface information into the deep layers by the feature model, Geosat data may miss some of the mesoscale features due to track separation. Other sources of error in the altimeter data are associated with temporal errors in the data. As seen in Fig. 4, the variability of mesoscale features in this region has a typical time scale of approximately 20 days, comparable to the 17-day repeat cycle of the Geosat satellite; thus, short-term temporal variations may be unresolved by the altimeter. The implication here is the obvious one that the use of the two data types should provide a better nowcast than if either of the two sets is used separately since each data type may fill some of the missing information associated with the other data type. For example, SST data may be used to identify eddies between altimeter tracks, and altimeter data may help to restore variability far from the Gulf Stream. Ezer et al. (1991), for example, suggested a possible interpolation scheme that combines altimetry and SST data to improve the nowcast skill compared to a case where only one data type is used (i.e., as in Mellor and Ezer 1991).

Although the small sample of synoptic SSH fields (nine) analyzed here is insufficient for a spatial and temporal spectral analysis diagnosis of the two data sources, the qualitative comparison in this study seems to partially contradict a conclusion of White et al. (1990) that spatial scales smaller than about twice the distance between satellite tracks $(\sim 300 \mathrm{~km})$ cannot be resolved by the Geosat altimeter data. Figure 3 clearly indicates that mesoscale eddies and the Gulf Stream meandering front are resolved quite well, though some eddies are missing from the Geosat data if they are located exactly between tracks.

The filtering effect of the statistical interpolation was studied, showing a reduction by as much as approximately $30 \%$ of the SSH variability (in some peaks) due to the interpolation. This reduction is more sensitive to the length-scale parameter than to the number of data points used to update each model grid point. This result may explain the underestimation of small-scale variability by statistical interpolation, as indicated by White et al. (1990) and Mellor and Ezer (1991).

This has been a comparative study of altimetry data and thermal analysis fields (derived from SST data) without the involvement of a dynamic model. It is, however, meant to be a step toward dynamic data assimilation, using a realistic numerical model of the North Atlantic Ocean. This will require some modifications to the interpolation scheme, since model and data errors are to be considered. A continuous data assimilation study with a three-dimensional optimal interpolation scheme based on the correlation technique of Mellor and Ezer (1991), and using Geosat 
altimetry data and OTIS analysis fields, is now under way.

Acknowledgments. This study is part of the Data assimilation and Model Evaluation Experiments (DAMEE) conducted by the Institute for Naval Oceanography (INO). The support of the INO under contract SC-S9256, and the additional support from the National Ocean Service (NOAA/NOS) through Sea Grant Project R/M-3P and from the Geophysical Fluid Dynamics Laboratory (NOAA/GFDL) through the use of their CRAY-YMP computer, are gratefully acknowledged.

\section{REFERENCES}

Blumberg, A. F., and G. L. Mellor, 1987: A description of a threedimensional coastal ocean circulation model. Three-Dimensional Coastal ocean Models, N. Heaps, Ed., Amer. Geophys. Union, $208 \mathrm{pp}$.

Cayula, J. F., and P. Cornillon, 1992: Edge detection algorithm for SST images. J. Atmos. Oceanic Technol., 9, 67-80.

Clancy, R. M., P. A. Phoebus, and K. D. Pollak, 1990: An operational global-scale ocean thermal analysis system. J. Atmos. Oceanic Technol., 7, 233-254.

- J. M. Harding, K. D. Pollak, and P. May, 1992: Quantification of improvements in an operational global-scale ocean thermal analysis system. J. Atmos. Oceanic Technol., 9, 55-65.

Cornillon, P., and D. R. Watts, 1987: Satellite thermal infrared and inverted echo sounder determination of the Gulf Stream northern edge. J. Atmos. Oceanic Technol., 4, 712-723.

Cummings, J. A., and M. J. Ignaszewski, 1991: The Fleet Numerical Oceanography Center regional ocean analysis system. MTS ' 91 , Proc. Marine Technology Society. New Orleans, 1123-1129.

Ezer, T., and G. L. Mellor, 1992: A numerical study of the variability and the separation of the Gulf Stream, induced by surface atmospheric forcing and lateral boundary flows. J. Phys. Oceanogr., 22, 660-682.
, and D.-S. Ko, 1991: Nowcasting the Gulf Stream structure with a primitive equation model and assimilation of altimetry and SST data. MTS '91. Proc. Marine Technology Society, New Orleans, 236-241.

- D.-S. Ko, and G. L. Mellor, 1992: Modeling and forecasting the Gulf Stream. Mar. Technol. Soc. J., 26, 5-14.

Glenn, S. M., D. L. Porter, and A. R. Robinson, 1991: A synthetic geoid validation of Geosat mesoscale dynamic topography in the Gulf Stream region. J. Geophys. Res., 96, 7145-7166.

Haines, B. J., G. H. Born, J. G. Marsh, and R. G. Williamson, 1990: Precise orbit computation for the Geosat exact repeat mission. J. Geophys. Res., 95, 2871-2886.

Holland, W. R., and P. Malanotte-Rizzoli, 1989: Assimilation of altimeter data into an ocean model: Space verses time resolution studies. J. Phys. Oceanogr., 19, 1507-1534.

Kelly, K. A., 1991: The meandering Gulf Stream as seen by the Geosat altimeter: Surface transport, position, and velocity variance from $73^{\circ}$ to $46^{\circ} \mathrm{W}$. J. Geophys. Res., 96, 16 721-16 738 .

Le Traon, P. Y., M. C. Rouquet, and C. Boissier, 1990: Spatial scales of mesoscale variability in the North Atlantic as deduced from Geosat data. J. Geophys. Res., 95, 20 267-20 286.

Levitus, S., 1982: Climatological atlas of the world ocean. NOAA Prof. Paper 13, U.S. Government Printing Office. 173 pp.

Mellor, G. L., and T. Ezer, 1991: A Gulf Stream model and an altimetry assimilation scheme. J. Geophys. Res., 96, 8779-8795.

- C. Mechoso, and E. Keto, 1982: A diagnostic calculation of the general circulation of the Atlantic Ocean. Deep-Sea Res., 29, 1171-1192.

Oey, L.-Y., T. Ezer, G. L. Mellor, and P. Chen, 1992: A model study of 'bump' induced western boundary current variabilities. $J$. Mar. Sys., 3, 321-342.

Robinson, A. R., M. A. Spall, L. J. Walsted, and W. G. Leslie, 1989: Data assimilation and dynamic interpolation in Gulfcast experiments. Dyn. Atmos. Oceans, 13, 301-316.

Sirkes, Z., and C. Wunsch, 1990: Note on apparent systematic and periodic errors in Geosat orbits. Geophys. Res. Lett., 17, 13071310.

White, W. B., C.-K. Tai, and W. R. Holland, 1990: Continuous assimilation of simulated Geosat altimetric sea level into an eddyresolving numerical ocean model. Part 1: Sea level differences. J. Geophys. Res., 95, 3219-3234. 